\title{
Transcription-related mutations and GC content drive variation in nucleotide substitution rates across the genomes of Arabidopsis thaliana and Arabidopsis lyrata Leah J DeRose-Wilson and Brandon S Gaut*
}

\author{
Address: Dept. of Ecology and Evolutionary Biology, U.C. Irvine, Irvine, CA 92697, USA \\ Email: Leah J DeRose-Wilson - Iderosew@uci.edu; Brandon S Gaut* - bgaut@uci.edu \\ * Corresponding author
}

Published: 23 April 2007

BMC Evolutionary Biology 2007, 7:66 doi:10.1 186/147|-2148-7-66

This article is available from: http://www.biomedcentral.com/I47/-2/48/7/66

(c) 2007 DeRose-Wilson and Gaut; licensee BioMed Central Ltd.

This is an Open Access article distributed under the terms of the Creative Commons Attribution License (http://creativecommons.org/licenses/by/2.0), which permits unrestricted use, distribution, and reproduction in any medium, provided the original work is properly cited.

\begin{abstract}
Background: There has been remarkably little study of nucleotide substitution rate variation among plant nuclear genes, in part because orthology is difficult to establish. Orthology is even more problematic for intergenic regions of plant nuclear genomes, because plant genomes generally harbor a wealth of repetitive DNA. In theory orthologous intergenic data is valuable for studying rate variation because nucleotide substitutions in these regions should be under little selective constraint compared to coding regions. As a result, evolutionary rates in intergenic regions may more accurately reflect genomic features, like recombination and GC content, that contribute to nucleotide substitution.
\end{abstract}

Results: We generated a set of 66 intergenic sequences in Arabidopsis lyrata, a close relative of Arabidopsis thaliana. The intergenic regions included transposable element (TE) remnants and regions flanking the TEs. We verified orthology of these amplified regions both by comparison of existing A. lyrata - A. thaliana genetic maps and by using molecular features. We compared substitution rates among the 66 intergenic loci, which exhibit $\sim 5$-fold rate variation, and compared intergenic rates to a set of 64 orthologous coding sequences. Our chief observations were that the average rate of nucleotide substitution is slower in intergenic regions than in synonymous sites, that rate variation in both intergenic and coding regions correlate with GC content, that GC content alone is not sufficient to explain differences in rates between intergenic and coding regions, and that rates of evolution in intergenic regions correlate negatively with gene density.

Conclusion: Our observations indicated that mutation rates vary among genomics regions as a function of base composition, suggesting that previous observations of "selective constraint" on non-coding regions could more accurately be attributed to a GC effect instead of selection. The negative correlation between nucleotide substitution rate and gene density provides a potential neutral explanation for a previously documented correlation between gene density and polymorphism levels within A. thaliana. Finally, we discuss potential forces that could contribute to rapid synonymous rates, and provide evidence to suggest that transcription-related mutation contributes to rate differences between intergenic and synonymous sites. 


\section{Background}

The primary processes that contribute to nucleotide substitution rates are mutation, selection, and population history, but their relative contributions can vary substantially among genes and genomic regions. For example, selection varies across genes as a consequence of protein function and gene expression patterns [1]. Similarly, mutation rates vary across genomic regions as a function of base composition and recombination rate [2,3]. Population history may be major determinant of substitution rate in the presence of selection, but should not be a factor in the absence of selection [4].

To date, our understanding about the evolutionary forces that contribute to nucleotide substitution rates has been based primarily on the study of coding regions. Inferences based on coding data reflect, in large part, the action of selection. An obvious example is variation in nonsynonymous substitution rates among genes, which is determined primarily by differential selective constraint. A subtler example is substitution rates at third codon positions, which are a function of both mutation and selection on codon usage. Highly biased genes evolve more slowly at synonymous sites [5-7], but synonymous substitution rates are also correlated with GC content [8-12]. The important point is that it can be difficult to disentangle the contribution of selection and mutation to rate variation among coding regions.

In contrast, non-coding regions should be under little selective constraint, and thus nucleotide substitution in these regions should be governed primarily by neutral processes like mutation. Studying non-coding regions can be difficult in practice, however, because orthology is not always clear. One way to identify orthologous non-coding regions is to compare, for example, non-coding regions that are 5' and 3 ' to orthologous genes [13]. The problem is that these regions are also expected to be enriched for functional elements, like promoter and enhancer sequences, and thus potentially under strong selection. Another way to identify orthologous regions is to compare non-coding regions among species via similarity (e.g., BLAST) searches, but this approach is also likely to enrich for slow-evolving regions under selective constraint. Indeed, selective constraint on non-coding regions may be more pervasive in eukaryote genomes than previously assumed [14]. For example, there is compelling evidence from Drosophila and several mammalian species that some non-coding regions evolve more slowly than synonymous sites [15], presumably due to selective constraint on non-coding nucleotide substitutions $[13,14,16]$. Thus, if non-coding data are not chosen carefully, it can be as difficult to disentangle the relative contribution of mutation and selection for non-coding data as it is for coding data.
To date, there have been few studies comparing evolutionary rates among plant nuclear genes $[17,18]$. The dearth of studies reflects, in part, difficulties substantiating orthology relationships, which are complicated because plant genomes contain more duplicated genomic regions than animal genomes [19]. This orthology problem is magnified for non-coding regions, which may evolve and rearrange more rapidly than coding regions. As a result, rates and patterns of sequence evolution among plant non-coding regions have not been characterized in any detail.

Here we contrast nucleotide substitution rates between orthologous coding and intergenic regions of Arabidopsis thaliana and Arabidopsis lyrata, two plant species that diverged $\sim 5$ million years ago [20]. Their genomes are largely collinear, but they differ in chromosome number (A. lyrata has eight chromosomes while A. thaliana has five; [21]), in DNA content (the A. lyrata genome contains $\sim 1.4 \mathrm{x}$ more DNA than that of $A$. thaliana; [22]), and by several translocations $[23,24]$. The two species also differ in population history; $A$. thaliana is predominantly selfing, while A. lyrata is an obligate outcrosser. These differences should affect differences in patterns and rates of molecular evolution between species, but the expected differences are not readily apparent [17], perhaps because A. thaliana only recently shifted to a selfing mating system [25].

To study rate variation in intergenic regions, we have generated sequence data in A. lyrata using PCR primers that flank remnants of transposable elements (TEs) in A. thaliana. We reason that these regions are non-functional by virtue of TE insertion, and thus comprise a data set that should be relatively free of selective constraint. We verify their orthology both with comparative maps of the two species and by their molecular features. The intergenic data are contrasted to a second data set consisting of large (> $400 \mathrm{bp}$ ) exonic sequences from A. lyrata and $A$. thaliana. With these two data sets, we address several questions about Arabidopsis nucleotide substitution rates, such as: $i$ ) do intergenic sequences evolve at rates similar to synonymous sites in coding data? ii) do any genomic features, like GC content or recombination, correlate with nucleotide substitution rate variation among loci? iii) what can be inferred about the relative contribution of mutation and selection to nucleotide substitution? and iv) do intergenic regions provide any hints to the mechanisms that contribute to genome size differences between A. lyrata and A. thaliana?

\section{Results \\ Isolation and location of orthologous intergenic regions}

We identified TE remnants in the A. thaliana genome and designed PCR primers to flank 576 of these remnants. Three primers were designed: two flanked the TE remnant, 
and a third (internal) primer was specific to the TE. We attempted amplification in A. lyrata with two separate PCR reactions for each of the 576 intergenic regions. The first used the two flanking primers, and the second used one flanking primer with the internal TE-specific primer. These two primer sets were also applied to A. thaliana ecotype Colombia as a positive control (data not shown). The intergenic regions were distributed across the A. thaliana genome and ranged in size from 200-2000 bases.

Of 576 attempted A. lyrata PCR amplifications, 34\% (198 of 576) were successful. Amplifications were considered a success when either of two cases occurred. In case one, the flanking primers amplified a band near the expected size and the internal primer also amplified a band of the expected size. The second successful case was when the flanking primers amplified a band consistent in size with the absence of the TE, and the internal primer failed to amplify any band. PCR failure was usually a failure of both primer combinations, but $\sim 10 \%$ of PCR "failure" was unexpected PCR results. Examples include patterns in which flanking primers amplified a band greatly different than the expected size or in which flanking primers amplified a small band consistent with the loss of the TE but amplification with the internal "TE" primer was successful.

In order to understand sampling biases associated with data generation, we mapped the location of PCR successes and failures along the genome to determine whether successful PCR amplifications were clustered with respect to their relative positions on the $A$. thaliana genome. We coded successful and unsuccessful PCRs as 1's and 0's, respectively, and created a binary string to represent the linear order of PCR results. We then calculated the variance in the number of zeros (failed PCRs) bounded by ones (successful PCRs) or the end of chromosomes. The observed variance in our data was compared to a distribution of variances based on 100,000 random shufflings of the binary string. The observed variance was much greater than $0.74 \%$ of simulated variances, indicating that successful PCR amplifications were significantly clustered ( $p$ $=0.0074)$. This clustering was related, in part, to gene density. We found that attempted amplifications were more likely to be successful in low gene density regions of $A$. thaliana (t-test; $p=0.027$ ), when we calculated gene density in a window of $0.50 \mathrm{Mb}$ around the A. thaliana TE. Consistent with this observation, all attempted amplifications were successful in regions with gene density lower than 90 annotated genes per $0.50 \mathrm{Mb}$, although there were only 5 amplifications in regions with density this low [see Additional File 1]. This effect seemed to be relatively local, however, because larger $1.0 \mathrm{Mb}$ windows retained the basic trend but the trend was no longer significant $(p=0.12)$.
Many of the amplicons were small, presumably due to the absence of the TE in A. lyrata. We cloned and sequenced a subset of 80 amplicons, yielding 66 alignable sequences. In order to help establish orthology, we examined the position of these 66 amplicons with respect to comparative maps of $A$. thaliana and A. lyrata $[23,24]$. We mapped each amplicon to the $A$. thaliana genome and found its place between markers that had been mapped on A. lyrata. We considered an amplicon as belonging to a collinear region of the genome if the two markers flanking the marker were also neighboring markers in A. lyrata. By this criterion, we could assign 45 of $66(68 \%)$ to unambiguously collinear regions. Another 20 were ambiguous either because they had only one flanking marker (i.e., they were at the end of a chromosome) or because a third marker had been rearranged between the two markers on the A. lyrata map. Only one amplicon was clearly in a noncollinear region. Although the resolution of comparative A. thaliana-A. lyrata maps is limited and the possibility of paralogy due to segmental duplication cannot be dismissed entirely, most $(68 \%)$ of our intergenic regions were orthologous by the criterion of collinearity.

The DNA sequence size of our 66 intergenic regions ranged from 218-1288 bp, with an average length of 732 bp [see Additional File 1]. None of the sequences had extensive open reading frames; the longest ORF was 111 bp. Based on the Arabidopsis small RNA project [26] targetfinder, there were also no obvious conserved small RNAs present in the sequences. Of the 66 regions, $49 A$. lyrata sequences contained a homolog of the TE remnant found in $A$. thaliana. These 49 sequences were alignable both in the TE remnant and in regions flanking the TE remnant, providing further molecular evidence for orthology. The remaining $17 \mathrm{~A}$. lyrata sequences did not contain the TE remnant, but the sequences were homologous to the regions flanking the A. thaliana TE. Altogether, 91\% (60 of 66) of our amplicons were either in a collinear region or contained the TE remnant with associated flanking regions, providing strong evidence that our amplicons are orthologs.

\section{Substitution rates in intergenic vs. synonymous sites}

Given orthologous sequences, we estimated evolutionary distances for intergenic loci. We report distances based on the K2P model ( $\left.d_{\mathrm{K} 2 \mathrm{P}}\right)$ [see Additional File 1 ], but all results and analyses are qualitatively identical using the general time-reversible model (data not shown). The average $d_{\mathrm{K} 2 \mathrm{P}}$ $\left(\bar{d}_{K 2 P}\right)$ estimate over all 66 regions was 0.105 substitutions per site (Figure 1 ). The $d_{\mathrm{K} 2 \mathrm{P}}$ estimates ranged $\sim 5$-fold from 0.042 to 0.228 substitutions per site. These numbers of course report the distances as if they were sampled from a single distribution, and a priori it might be thought (for 
example) that sequences with TEs evolve at different rates than those without. There is, however, no evidence to this effect (t-test, $p=0.57$; Mann-Whitney U, $p=0.91$ ). Similarly, we examined whether evolutionary rates differed by collinearity, reasoning that paralogous sequences could be represented in the group for which collinearity was ambiguous. There was no difference between groups based on collinearity (t-test, $p=0.81$; Mann-Whitney U, $p$ $=0.34$ ). Furthermore, the six amplicons for which there was no evidence for orthology from either TE presence or collinearity fell well within the extremes of the $d_{\mathrm{K} 2 \mathrm{P}}$ range ( $d_{\mathrm{K} 2 \mathrm{P}}$ ranges from 0.074 to 0.120 for these six; [see Additional File 1]). Because there is no obvious evidence for rate classes based on TE presence or collinearity, we treated the 66 intergenic sequences as a single group.

In addition to our non-coding data set of 66 sequences, we generated an additional set of 64 large (400-700 bp) exons. These exons were distributed throughout the genome [see Additional File 2]. Forty of these 64 genes are located in non-ambiguously collinear regions between $A$. thaliana and A. lyrata. Of the remaining 24, 23 had ambiguous collinearity and one was in a non-collinear region. We calculated synonymous distances $\left(d_{\mathrm{s}}\right)$ between sequences for all 64 loci. The 24 genes with uncertain col-

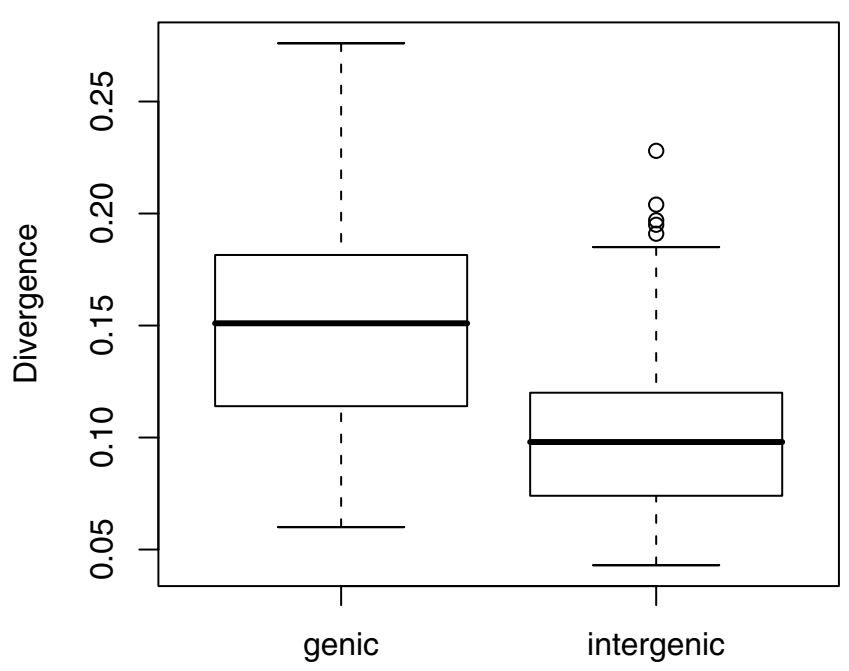

Type

\section{Figure I}

A box plot of genetic distances in the two sequence classes: intergenic and coding. The box represents the interquartile range, with the lines extending the range of the data. Points outside the range are mild outliers, with values greater then $\mathrm{I} .5 \mathrm{X}$ the upper bound of the interquartile range. linearity did not differ from the remaining 40 loci either as group (t-test, $p=0.80$; Mann-Whitney $U, p=0.85$ ), but two of these exons had the highest $d_{\mathrm{s}}$ values in our sample, with one value $>1.5$-fold higher than that of any other exon [see Additional File 2]. We chose to remove these two loci from further analyses because they may represent paralogs, although none of our overall results vary with their inclusion. Based on the remaining 62 exons, mean estimates of $d_{\mathrm{s}}\left(\bar{d}_{\mathrm{s}}\right)$ were 0.148 substitutions per synonymous site, with a range from 0.060 to 0.259 (Figure 1 ).

Our principle goal is to compare substitution patterns and rates between coding and intergenic regions. Loci within the two data types varied $\sim 5$-fold in genetic distance. The coefficients of variation, which was 0.41 for intergenic data and 0.33 for coding data, indicate that distances vary to similar degrees for the two data types. However, the mean genetic distance differed between the two classes (Figure 1), with synonymous sites evolving more rapidly than non-coding sites ( $t$-test, $p<0.001$ ). Mean distances can be used to estimate nucleotide substitution rates. Assuming that the two lineages diverged roughly 5 million years ago [20], the mean substitution rate for synonymous sites was $1.55 \times 10^{-8}$ substitutions per site per year. In contrast, the mean non-coding rate was only two-thirds of that value, at $1.05 \times 10^{-8}$ substitutions per site per year (Figure 1).

\section{Genomic correlates with substitution rates}

Our results indicate that mean genetic distances vary between data types and also that genetic distances vary among loci. We also sought to determine whether genetic distances are related to genomic features such as GC content and recombination rate. We expect that such relationships will be clearer in intergenic data, because the patterns are less likely to be complicated by selective forces.

Recombination may contribute to rate variation among loci by introducing mutations $[27,28]$. Recombination rates have been estimated for $A$. thaliana, based on comparisons of physical (Mega bases) and genetic (centiMorgan) distances $[29,30]$, but there are no direct estimates of $\mathrm{cM} / \mathrm{Mb}$ recombination rates for $A$. lyrata. We therefore used $A$. thaliana recombination rates to investigate relationships between recombination rate and genetic distances. There was no strong correlation between recombination rate and genetic distance whether the data were combined ( $r=0.10$; Figure 2$)$ or examined separately as intergenic and genic.

The lack of correlation between recombination rates and genetic distances parallels a previous study that detected no correlation between recombination rates and $A$. thal- 


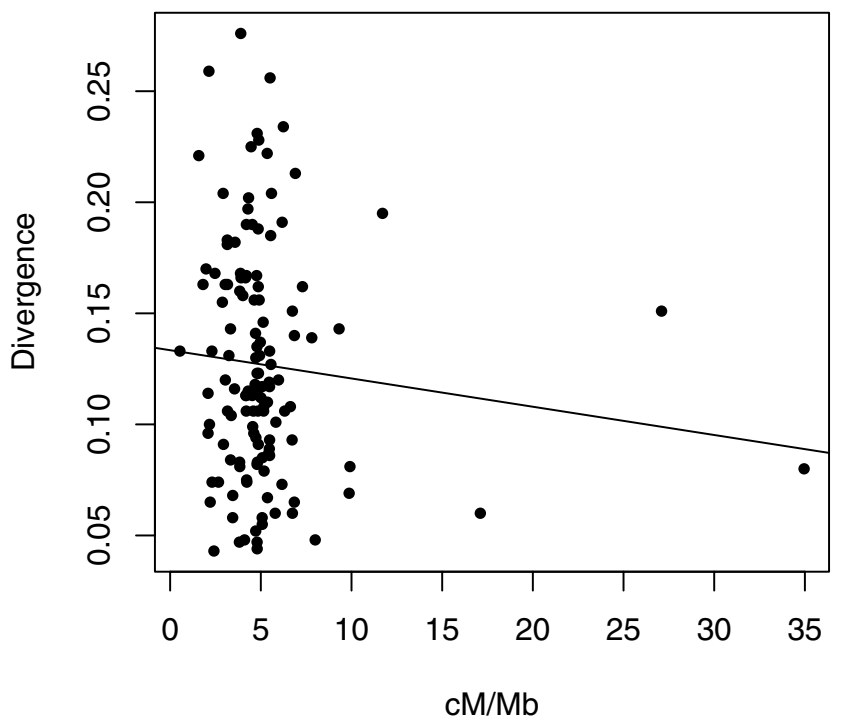

Figure 2

The correlation between recombination rate ( $x$-axis) and genetic distance is not significant for combined coding and non-coding data $(r=-0.10)$. Filled circles represent coding loci, and empty circles are non-coding loci.

iana sequence polymorphism [31]. However, Nordborg et al. (2005) did detect a negative correlation between polymorphism and gene density. Given this observation, we too examined the relationship of genetic distance with gene density. Gene density is negatively correlated with substitution rate in both datasets, although this relationship is only significant in the intergenic data $(r=-0.33 ; p$ $=0.086)$ and not in coding data $(r=-0.1 ; p=0.29)$ (Figure 3 ). These results were generated with gene density measured as the number of genes in a $0.50 \mathrm{Mb}$ window centered around each intergenic locus; the result is similar, but only borderline significant, with $1.0 \mathrm{Mb}$ windows $(r=$ $-0.18 ; p=0.09$ ).

GC content is another factor known to correlate with substitution rate. The GC content of our intergenic sequences did not differ markedly between $A$. lyrata and A. thaliana (37.9 and $37.4 \%$ GC, respectively). Genic regions also had similar GC content between the two species, but average GC values for coding DNA were $7.3 \%$ higher than intergenic regions $(44.9 \%$ vs. $37.6 \%$, respectively). This contrast also held when only synonymous sites in coding regions were considered ( $43.1 \%$ vs. $37.6 \%$, respectively). The observed transition:transversion ratio was also higher for coding data (1.73) than for intergenic regions (1.36), although statistical support for this trend was borderline (G-test, $p=0.09$ ). CpG deamination is a common explanation for elevated transition:transversion ratios in high GC regions and could cause differences in rates between

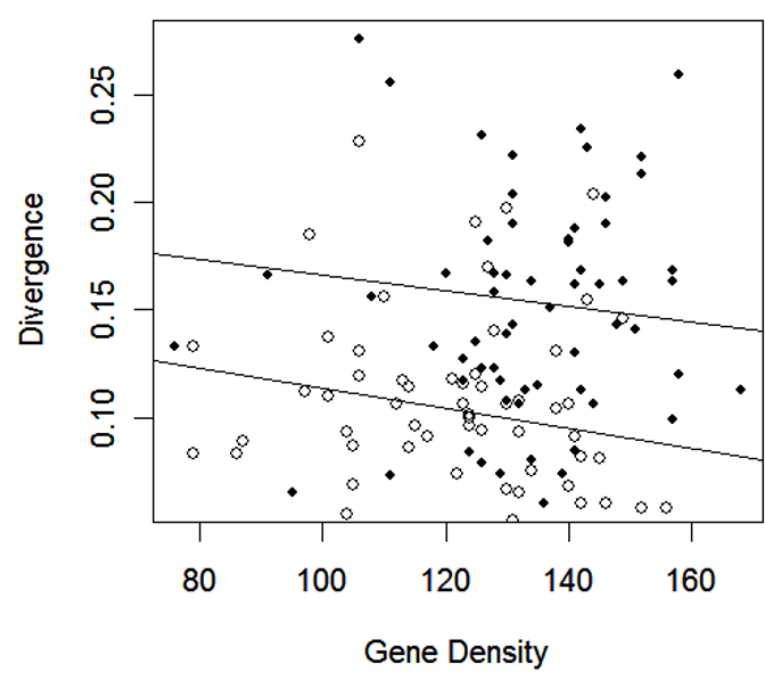

Figure 3

The correlation between gene density, based on the number of genes in a $0.5 \mathrm{Mb}$ window, and divergence is negative for both coding and non-coding data. Filled circles represent coding loci, and empty circles are non-coding loci. The higher regression line is based on coding data.

genic and intergenic regions. We thus recalculated divergence, treating all $\mathrm{CpG}$ dinucleotides as non-variable in both coding and intergenic data sets. Although there were more $\mathrm{CpG}$ sites in genic regions, the $\mathrm{CpG}$ disparity alone did not explain the difference in rate between genic and intergenic regions. Genic synonymous divergence rates without CpG sites was $1.22 \times 10^{-8}$ substitutions per site per year, while intergenic divergence rates were $0.80 \times 10^{-}$ ${ }^{8}$ substitutions per site per year. These rates are still significantly different from each other $(\mathrm{p}<0.001)$.

With combined intergenic and genic data, there was a strong positive correlation between genetic distance and GC content $(r=0.35 ; p<0.0001$; Figure $4 \mathrm{~A})$. This correlation also held for synonymous sites alone $(r=0.33 ; p<$ 0.001 ). To better investigate the relationship between GC and genetic distances, we performed an analysis of covariance. The ANCOVA examined the effect of GC on genetic distance, with sequence type as a factor to determine if GC content alone accounted for the differences in genetic distance between our coding and intergenic data (Figure 4B). The ANCOVA model indicated that GC content is a significant predictor for genetic distance $(p=0.003)$, but also that there is an additional effect of sequence type ( $p=$ $0.001)$.

\section{Patterns of indel variation}

Intergenic regions do not have constraints on coding frame, and thus accumulate indels. These indels, in turn, 

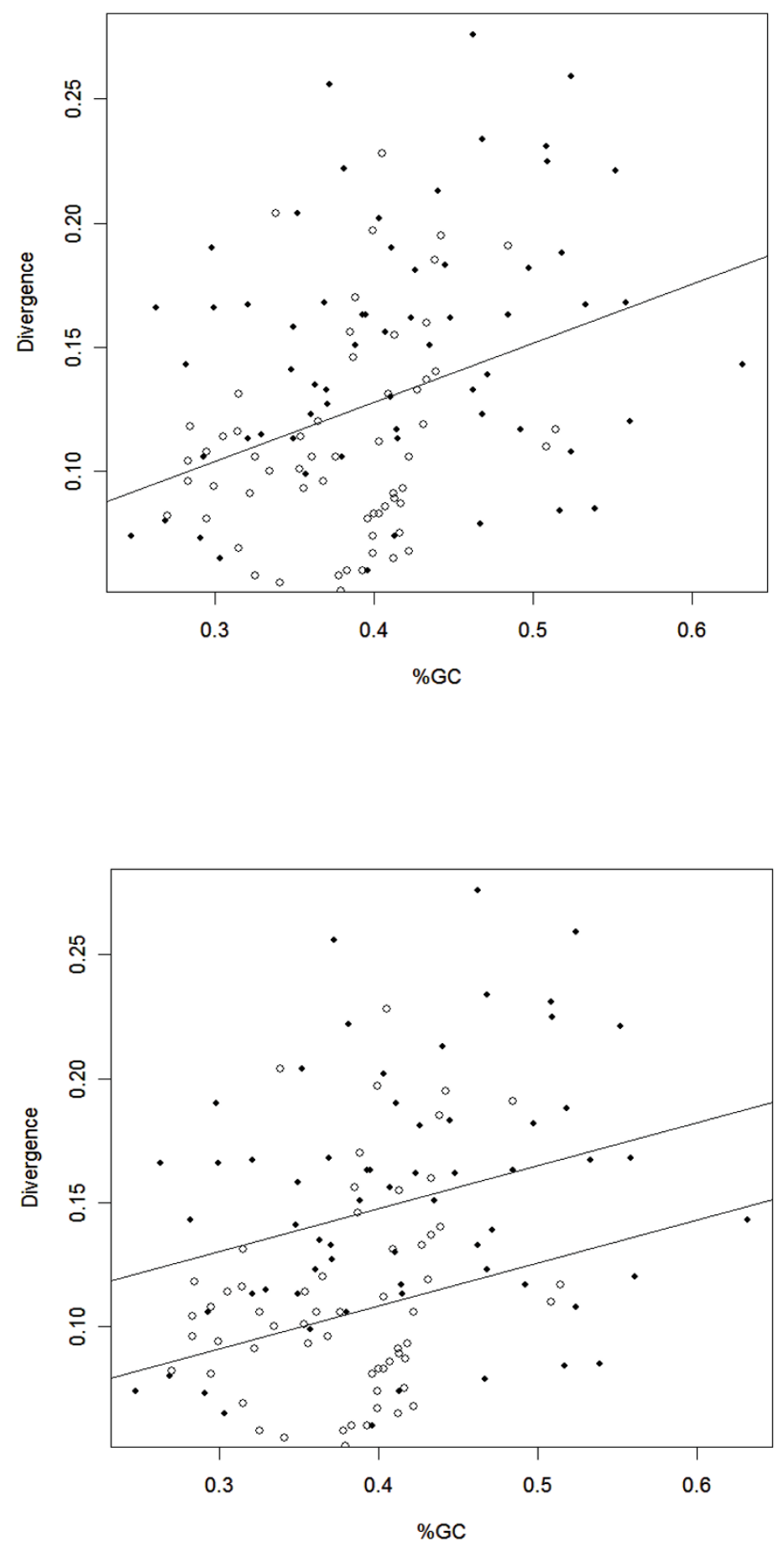

\section{Figure 4}

A) The correlation between GC content and genetic distance across both data types $(r=0.35 ; p<0.000 \mathrm{I})$. B) Analysis of covariance with sequence type, GC content and genetic distance. GC content contributes significantly $(p<0.003)$ to the variance in divergence, but there is an additional effect of sequence type on genetic distance that is not accounted for by GC content ( $p$ $<0.00 \mathrm{I})$. For both graphs, filled circles represent coding data and empty circles represent intergenic data. 
may provide insights into processes that contribute to the $1.4 \times$ size difference between $A$. lyrata and $A$. thaliana genomes. We analyzed indel size distributions in intergenic data. For these analyses, we ignored sequences in which the remnant TE was present in $A$. thaliana but absent in A. lyrata. We also ignored gaps at sequence ends. For the purpose of clarity, we denoted the species with the non-gapped sequence in an alignment as containing an 'insertion'. (In point of fact, one cannot determine whether a gap is due to an insertion or a deletion without the benefit of an outgroup.)

Our intergenic data contained 267 distinct $A$. lyrata insertions totaling 1565 bases. A. thaliana, had more distinct insertions (321) but fewer inserted bases (1499). The mean size of insertions was 5.9 bp in A. lyrata and 4.7 bp in A. thaliana, and did not differ significantly. In general, A. lyrata insertions tended to be longer and fewer (Figure 5). Although the distributions of insertion sizes appear somewhat different, with the A. lyrata distribution having a longer tail of large insertions and $A$. thaliana having a higher proportion of small insertions, statistical tests com- paring the distributions of the samples were not significant (Kolmogorov-Smirnov; $p=0.13$ ). Furthermore, a sign test comparing the relative length of $A$. thaliana and A. lyrata sequences across loci was not significant $(p=$ 0.34 ), and thus there is no evidence that intergenic regions are systematically longer in A. lyrata.

\section{Discussion}

We have generated a sample of orthologous intergenic sequences from A. lyrata to compare to A. thaliana. These sequences were designed to include remnants of transposable elements (TEs), with the a priori thought that these regions are not under strong selection and therefore that the distribution of evolutionary rates provides insight into neutral genomic processes. Nonetheless, our analyses of the genomic location of PCR successes reveal subtle biases in our non-coding data that are important to keep in mind when discussing nucleotide substitution rates. First, the data are not random with respect to genomic location and thus may be biased toward more slowly evolving regions of the genome. Second, they tend to originate from regions of low gene density. This does superficially sug-

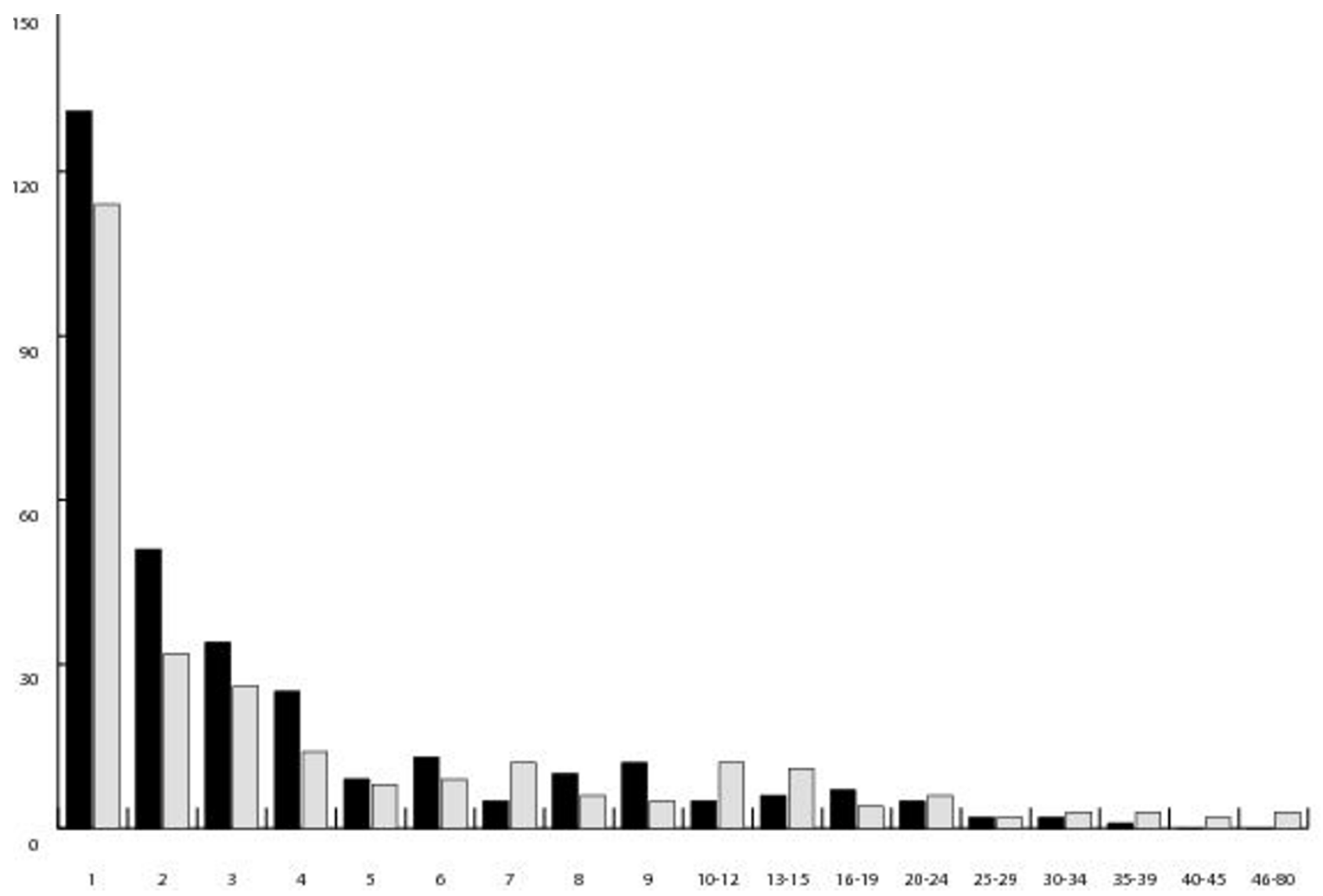

Figure 5

Distribution of insertion sizes in A. lyrata and A. thaliana intergenic regions. A. thaliana insertions are presented in black, $A$. lyrata in grey. 
gest, however, that they are not enriched for functional elements like enhancers or promoters. Third, most of the sequences originate from regions of genome collinearity. Coupled with molecular features, there is strong evidence that our final data set of 66 non-coding regions represents orthologous DNA features. This set of non-coding sequences represents one of the few - and perhaps only multi-locus, intergenic divergence data in plants.

We compared our intergenic data to 64 long exons that were sequenced in A. lyrata. The primary result is that the mean rate of intergenic nucleotide substitution is twothirds that of the synonymous coding data, with an absolute rate estimated to be $1.05 \times 10^{-8}$ substitutions per site per year. This result holds with alternative nucleotide models (see Methods), and thus does not appear to be solely an issue of estimation procedures.

Slower rates in non-coding regions relative to synonymous sites are becoming a surprisingly frequent observation. For example, a recent study of Drosophila demonstrated that non-coding DNA evolves considerably slower than synonymous sites in terms of both divergence between species and polymorphism within species [16]. By comparing studies, one can also make the case that pseudogenes [32,33] and introns [34,35] evolve more slowly than synonymous sites in apes and other mammals [13,36-38]. Studies of mammalian intergenic regions have also found slower rates than synonymous sites $[35,39,40]$. Although most of these studies encompass only a handful of genes, an overall picture of relatively slow non-coding rates is emerging.

Why are non-coding rates slow relative to synonymous rates? One potential reason discussed above (see Introduction) is methodological biases. Our observations were consistent across three different alignment methods and were qualitatively similar when we performed the analyses on synonymous and four-fold degenerate sites using the same K2P model as we applied to intergenic data. Thus, neither model choice nor (mis)alignment appears to contribute substantially to our inferences. Nonetheless, one cannot fully discount sampling biases in the noncoding data, particularly because only $34 \%$ of our intergenic PCR amplifications were successful. Some of this failure is attributable to the fact that some TEs, particularly Basho elements, do not appear to be shared between the two species and thus could not be amplified in A. lyrata. We attempted to evaluate the magnitude and effect of PCR bias by performing a parallel experiment on coding data. We designed three primers to amplify each of the 64 exons, with the outside primers anchored in flanking intronic or UTR regions and an internal primer specific to the exon [see Additional File 2]. To mimic our amplifications of noncoding DNA, we applied the same PCR proce- dures and same criteria as we applied to noncoding data (see Methods). Although 62 of 64 amplifications were successful in A. thaliana, only 25 of 64 (39\%) were successful in A. lyrata, roughly mimicking the success rate (34\%) of noncoding amplifications. The group of exons that were successfully amplified by this method did not differ from "unsuccessful" loci in mean genetic distance (0.154 vs. 0.155, respectively; [see Additional File 2], indicating that PCR success did not heavily bias the inference of genetic distance and substitution rates. If this result is general, then our observation of slow noncoding rates is not solely a function of amplification biases.

A second reason for slow evolutionary rates is selection. Andolfatto (2005) has concluded that non-coding regions of Drosophila are under selective constraint and also subject to bouts of adaptive selection. It seems unlikely that this is a general phenomenon, however, particularly for plants. Plant genomes change rapidly in size, in large part due to the accumulation of repetitive DNA [41]. It is thus difficult to imagine that this repetitive DNA is under strong selective constraint. Arabidopsis species may be an exception because they have relatively little repetitive DNA for plant genomes. Nonetheless, there is little evidence for selection on non-coding regions that flank coding DNA [42]. Further, the selective interpretation is particularly difficult to argue in this case because the intergenic regions in our study were a priori chosen for their apparent lack of function and tend to originate from genomic regions of low gene density. Consistent with the possibility of low functionality, the intergenic data were replete with indel variation. A previous study found that intron lengths from 22 genes differed significantly between these two species [17], suggesting that the $1.4 \times$ difference in genome size between A. lyrata and A. thaliana is due, at least in part, to the accumulation of small sequence changes. Surprisingly, intergenic indel patterns and lengths do not differ substantially between A. lyrata and $A$. thaliana, and we find no evidence that differential indel events contribute to differences in genome size.

The third explanation for relatively slow rates of evolution in non-coding DNA is differential mutation rates. Recombination could contribute to this mutational effect, because recombination can cause mutations during the resolution of double-strand breaks [43]. If recombination and mutation are associated processes and most mutations are neutral, then both polymorphism and divergence should be positively correlated with recombination rate. However, we find no correlation between genetic distances and A. thaliana recombination rates (Figure 2). Similarly, recombination does not correlate with polymorphism in 1000 A. thaliana gene fragments [31] or 26 A. lyrata genes [44]. It is possible, of course, that the lack of correlation between evolutionary rate and recombina- 
tion is a false negative. A true positive correlation might go undetected if recombination rate estimates are imprecise (see [45]) or if there have been shifts in genomic patterns of recombination between $A$. thaliana and A. lyrata. To date, there is little evidence for the latter $[23,24,44,46]$. Overall, we have no evidence to suggest that recombination rates contribute to the differences in rates between intergenic and genic DNA or to the observed $\sim 5$-fold variation in evolutionary rates among loci.

In contrast to recombination rates, there is a strong relationship between genetic distance and GC content in our data (Fig. 3). Substitution rates have long been known to correlate positively with GC content $[10,28,47-49]$, presumably due to higher mutation rates in high GC regions, in part due to CpG effects $[8,48,50]$. Consistent with these previous observations, the synonymous sites of our coding data have a high average GC content relative to intergenic data, faster evolutionary rates, and a higher proportion of transitional (as opposed to transversional) changes. Interestingly, GC could contribute to some of the non-coding effects observed in Drosophila, too. There is a striking difference in GC content between the coding and non-coding regions analyzed by Andolfatto (2005); noncoding sequences have a mean GC content of $43 \%$, compared to $58 \%$ GC in coding regions. This disparity alone, rather than selective constraint, may account for the slow rate of divergence in Drosophila non-coding DNA. GC content alone cannot explain, however, the skewed ratios of polymorphism to divergence found by Andolfatto (2005), unless there has been a recent shift in GC mutation biases.

Nonetheless, GC content does not provide a complete explanation for evolutionary rate variation in our data. One of the most interesting aspects of genome-wide analyses in A. thaliana has been the negative correlation between gene density and polymorphism in A. thaliana [31]. This correlation has been interpreted to be the consequence of either selective sweeps or, more likely, background selection $[31,51]$. We have uncovered a similar negative correlation between gene density and evolutionary rate for intergenic data (Figure 3), providing a potential neutral explanation for the observation - i.e., that mutation rates are higher in regions of low gene density. Note also that, if anything, our intergenic data are biased to low gene density regions of the genome where hitchhiking and background selection should not be particularly strong. The negative correlation between genetic distance and gene density remains borderline significant with partial correlations that also consider GC content $(r$ $=-0.21, p=0.08$ ). Thus, the gene density effect does not appear to be solely an issue of GC, but the causes of this effect are elusive at this point.
More importantly, there is a "sequence-type" difference between genic and intergenic substitution rates that is not accounted for by differences in GC content (Figure 4B). Differential selection of genomic regions is unlikely to explain the observed differences between sequence types, as we would expect the substitution rate effect to be in the opposite direction (Figure 1). It is possible, though, that mutation varies between intergenic and coding regions. For example, transcription-related mutations could increase synonymous substitution rates in coding regions over and above GC effects. This possibility seems plausible because A. thaliana base composition varies as a function of gene expression, suggesting that mutation rates among genes scale with transcription rates [42]. If this hypothesis is correct, then introns should evolve at a rate that is more similar to exons than to intergenic regions. To examine this hypothesis, we aligned the 29 available $A$. lyrata introns from Genbank to their A. thaliana ortholog. The mean distance $\left(\bar{d}_{K 2 P}\right)$ between intron sequences was 0.157 substitutions per site. This distance does not differ significantly from our synonymous divergence estimates ( $p=0.34$ ), but it is significantly higher than the mean genetic distance at our 66 intergenic loci $(p=0.006)$. Thus, although the data are limited, intronic sequences are consistent with the hypothesis that transcription-related mutation contributes to differential substitution rates between exons and intergenic regions.

\section{Conclusion}

It is clear that GC content is a major determinant of evolutionary rate variation, not only between sequence types (intergenic and coding) but also among loci. GC content may contribute to $A$. thaliana polymorphism, too, because there is a positive, borderline significant correlation $(r=$ $0.21 ; p=0.08$ ) between GC content and SNP polymorphism (as measured by $\pi$, the average pair wise difference among a sample of sequences) for 140 polymorphic intergenic loci in the Nordborg et al. (2005) data panel of 96 individuals (data not shown). On the other hand, GC content does not fully explain intergenic rate variation; variation also correlates with gene density after correcting for GC content. More importantly, there is a detectable effect of sequence type. The explanation that we deem most reasonable for contributing to the sequence-type effect is transcription-related mutation. Transcriptionrelated mutation predicts a pattern of higher evolutionary rates in transcribed regions, and this prediction is upheld with both exonic and intronic data. 


\section{Methods \\ Sequence data}

To isolate and sequence orthologous non-coding regions, we started by identifying TEs in the A. thaliana genome with a BLASTn search, using a 1 e-20 cut-off and no repeat filtering, against the release 4 genome. The TE queries in this search were tabulated from three sources: $i)$ TEs described in a previous survey of $17 \mathrm{Mb}$ of the A. thaliana genome [52]; ii) A. thaliana TEs found in TIGR's repeat database; and iii) all GenBank ORFs annotated as transposase-related in the Arabidopsis genome release 4.0. In this search, we identified 3,079 non-redundant TE sequences ranging in length from $65 \mathrm{bp}$ to $15.8 \mathrm{~kb}$, with a mean length of 1,134 bases.

We designed primers to flank 576 of these TE remnants. Primers were based on $A$. thaliana genomic sequence using primer3 with default parameters. Three primers were designed: two flanked the TE remnant, and a third (internal) primer was specific to the TE. We attempted amplification in A. lyrata with two separate PCR reactions for each of the 576 intergenic regions. The first used the two flanking primers, and the second used one flanking primer with the internal TE-specific primer. These two primer sets were also applied to A. thaliana ecotype Colombia as a positive control (data not shown). PCR was performed with a 58/51 touchdown protocol with 1 minute denaturing at $95^{\circ} \mathrm{C}, 45$ second annealing at $58^{\circ} \mathrm{C}$, and 1.5 minute extension at $70^{\circ} \mathrm{C}$ for 15 cycles, followed by 10 cycles of 1 minute denaturing at $95^{\circ} \mathrm{C}, 45$ second annealing at $51^{\circ} \mathrm{C}$, and 1.5 minute extension at $70^{\circ} \mathrm{C}$, and completed with a 7 min elongation period at $70^{\circ} \mathrm{C}$. Plant material for all A. lyrata PCR and sequence data was extracted from a single Icelandic individual (provided by S. Wright, York University). DNA extraction employed the DNeasy plant mini kit with the standard protocol.

PCR amplicons were cloned using the pGem-T Easy vector system. Amplicons with multiple bands were gel purified before cloning. In total, 198 regions were successfully amplified in A. lyrata. Only a subset of these were cloned and sequenced, however, since many of the amplified regions apparently did not contain TEs in A. lyrata and thus the amplified regions were quite small. Additionally some intergenic regions were larger then could be readily cloned. The clones were sequenced using the standard ABI sequencing protocol with BigDye 3.1 terminator kit, and sequences were visualized on an ABI 3100. For most amplicons only a single clone was sequenced. However, a set of eight intergenic regions was cloned and sequenced five times to estimate error from cloning and sequencing. The error rate was estimated to be $\sim 2.5 \times 10^{-4}$ errors per site. At this rate, error contributed only $\sim 2 \%$ uncertainty to our distance estimates between species, and we thus disregarded error in subsequent analyses. Intergenic sequence data were submitted to Genbank [see Additional File 1].

We also amplified and sequenced a set of 64 exons from the same Icelandic accession used to isolate intergenic regions. As explained in Wright et al. (2006), each exon was submitted to a BLAST search [53] against the shotgun genome sequence of Brassica oleracea. Homologous $B$. oleracea regions were aligned to $A$. thaliana data to identify conserved regions for primer design. PCR primers were designed with PrimerQuest (Integrated DNA Technologies). Primers and exons were also submitted to a BLAST search against the A. thaliana genome to ensure singlecopy status. The primers, as well as the list of loci, are provided in Additional File 2 and also described in Wright et al., (in prep). Exon amplifications were based on PCR conditions that included 30 cycles of 30 second denaturing at $95^{\circ} \mathrm{C}, 45$ seconds annealing at $55^{\circ} \mathrm{C}$, and $1 \mathrm{~min}$ extension at $70^{\circ} \mathrm{C}$. Amplification products were sequenced directly using ABI BigDye 3.1 and the ABI 3100 automated sequencer. Bases were called using Phred and Phrap. Heterozygotes were resolved with Polyphred [54] and extensive manual trace examination. Only one haplotype was used per locus for analysis. The data are available in Genbank [see Additional File 2].

\section{Alignments}

Nucleotide alignments for both intergenic and coding sequences were generated with ClustalW [55], using default parameters. Indels in the intergenic sequences created some ambiguity in a subset of the alignments. These alignments were inspected manually and in cases where there was a clear resolution the alignments were adjusted manually with Bioedit. Because of our concern about alignment ambiguity bordering indels, we generated a second alignment set for the intergenic data, eliminating eight bases on either side of each indel. We also aligned all intergenic sequences using mcalign2, a program specifically for alignments of intergenic sequences containing indels [56]. This program is optimized for Drosophila indel patterns, and parameters for Arabidopsis deletions are not known. However, mcalign 2 alignments were nearly identical to the original alignments. We also eliminated 14 sequence alignments from analyses either because extensive indels made confident alignments impossible or because a substantial portion of the A. lyrata sequence did not appear to be homologous to the $A$. thaliana sequence. Sequence alignments are available from [57].

\section{Sequence analyses}

Genetic distances between A. lyrata and A. thaliana intergenic sequences were estimated using the Kimura 2parameter (K2P) model implemented in Mega 2.0. Coding frames for determining synonymous sites was established from A.thaliana gene annotations. The Nei- 
Gojobori method implemented in MEGA2.0 [58] was used to estimate $\mathrm{K}_{\mathrm{S}}$ in the coding sequences. Genetic distances for coding regions were also calculated using only four fold degenerate sites and implementing the K2P model. Changing models did not affect the overall mean divergence estimates, and the original estimates based on the Nei and Gojobori model are presented in all analyses. Recombination rate estimates were based on a previous analysis of $A$. thaliana [30].

\section{Authors' contributions}

LJDW and BSG designed the experiments and analyses and wrote the paper; LJDW performed experimental work and analyses. Both authors read and approved the final manuscript.

\section{Additional material}

\section{Additional file 1}

Supplemental information for intergenic sequences. Information, including genetic distance estimates, primer sequences and genbank IDs, for each intergenic sequence analyzed.

Click here for file

[http://www.biomedcentral.com/content/supplementary/14712148-7-66-S1.xls]

\section{Additional file 2}

Supplemental information for genic sequences. Information, including genetic distance estimates, primer sequences, gene identifiers, genbank IDs and other information for each genic sequence analyzed.

Click here for file

[http://www.biomedcentral.com/content/supplementary/14712148-7-66-S2.xls]

\section{Acknowledgements}

The authors want to thank S. Wright, N. Galtier and two anonymous reviewers for comment and discussion. This work supported by NSF grant DEB-0426166.

\section{References}

I. Duret L, Mouchiroud D: Determinants of Substitution Rates in Mammalian Genes: Expression Pattern Affects Selection Intensity but Not Mutation Rate. Mol Biol Evol 2000 I 7(I):68-70.

2. Hellmann I, I E, SE P, S P, M P: A neutral explanation for the correlation of diversity with recombination rates in humans. American Journal of Human Genetics 2003, 72(6): I527-I535.

3. Spencer CCA, Deloukas P, Hunt S, Mullikin J, Myers S, Silverman B, Donnelly P, Bentley D, McVean G: The Influence of Recombination on Human Genetic Diversity. PLoS Genetics 2006, 2(9):el 48.

4. Kimura M: Evolutionary Rate at the Molecular Level. Nature 1968, 21 7:624-626.

5. Akashi H: Synonymous Codon Usage in Drosophila melanogaster: Natural Selection and Translational Accuracy. Genetics 1994, I 36(3):927-935.

6. Sharp PM, Li WH: The rate of synonymous substitution in enterobacterial genes is inversely related to codon usage bias. Mol Biol Evol 1987, 4(3):222-230.

7. Eyre-Walker A, Bulmer M: Synonymous Substitution Rates in Enterobacteria. Genetics 1995, I40(4): |407-|4|2.
8. Zavolan M, Kepler TB: Statistical inference of sequencedependent mutation rates. Current Opinion in Genetics \& Development 200I, I I(6):6I2-6I5.

9. Wolfe $\mathrm{KH}$, Sharp $\mathrm{PM}, \mathrm{Li} \mathrm{WH}$ : Mutation rates differ among regions of the mammalian genome. Nature 1989, 337(6204):283-285.

10. Hurst LD, Williams EJB: Covariation of GC content and the silent site substitution rate in rodents: implications for methodology and for the evolution of isochores. Gene 2000, 26 I(I): $107-1 \mid 4$

II. Gu X, $\mathrm{Li} \mathrm{WH:} \mathrm{The} \mathrm{size} \mathrm{distribution} \mathrm{of} \mathrm{insertions} \mathrm{and} \mathrm{deletions}$ in human and rodent pseudogenes suggests the logarithmic gap penalty for sequence alignment. Journal of Molecular Evolution 1994, 40(4):464-473.

12. Bernardi G, Olofsson B, Filipski ], Zerial M, Salinas J, Cuny G, MeunierRotival M, Rodier F: The mosaic genome of warm-blooded vertebrates I0.1 I26/science.4001930. Science 1985 228(4702):953-958

13. Bush EC, Lahn BT: Selective Constraint on Noncoding Regions of Hominid Genomes. PLoS Computational Biology 2005, I(7):e73.

14. Drake JA, Bird C, Nemesh J, Thomas DJ, Newton-Cheh C, Reymond A, Excoffier L, Attar H, Antonarakis SE, Dermitzakis ET, Hirschhorn JN: Conserved noncoding sequences are selectively constrained and not mutation cold spots. Nat Genet 2006, 38(2):223-227

15. Dermitzakis ET, Reymond A, Scamuffa N, Ucla C, Kirkness E, Rossier C, Antonarakis SE: Evolutionary Discrimination of Mammalian Conserved Non-Genic Sequences (CNGs). Science 2003, 302(5647): $1033-1035$.

16. Andolfatto P: Adaptive evolution of non-coding DNA in Drosophila. Nature 2005, 437(7062): I|49-II 52.

17. Wright SI, Lauga B, Charlesworth D: Rates and Patterns of Molecular Evolution in Inbred and Outbred Arabidopsis. Mol Biol Evol 2002, I 9(9): |407-| 420.

18. Zhang L, Vision TJ, Gaut BS: Patterns of Nucleotide Substitution Among Simultaneously Duplicated Gene Pairs in Arabidopsis thaliana. Mol Biol Evol 2002, I9(9):|464-|473.

19. Lockton S, Gaut BS: Plant conserved non-coding sequences and paralogue evolution. Trends in Genetics 2005, 2 I ( I):60-65.

20. Koch MA, Haubold B, Mitchell-Olds T: Comparative Evolutionary Analysis of Chalcone Synthase and Alcohol Dehydrogenase Loci in Arabidopsis, Arabis, and Related Genera (Brassicaceae). Mol Biol Evol 2000, I 7( I 0): |483-| 498.

21. Jones BMG: Experimental taxonomy of the genus Arabis. Leicester, UK , University of Leicester,; 1963.

22. Johnston JS, Pepper AE, Hall AE, Chen ZJ, Hodnett G, Drabek J, Lopez $\mathrm{R}$, Price HJ: Evolution of Genome Size in Brassicaceae. Ann Bot 2005, 95(I):229-235.

23. Yogeeswaran K, Frary A, York TL, Amenta A, Lesser AH, Nasrallah JB, Tanksley SD, Nasrallah ME: Comparative genome analyses of Arabidopsis spp.: Inferring chromosomal rearrangement events in the evolutionary history of A. thaliana. Genome Res 2005, I 5(4):505-5। 5.

24. Kawabe A, Hansson B, Hagenblad J, Forrest A, Charlesworth D: Centromere Locations and Associated Chromosome Rearrangements in Arabidopsis lyrata and A. thaliana. Genetics 2006, I73(3): 1613-1619.

25. Shimizu KK, Cork JM, Caicedo AL, Mays CA, Moore RC, Olsen KM, Ruzsa S, Coop G, Bustamante CD, Awadalla P, Purugganan MD: Darwinian Selection on a Selfing Locus. Science 2004, 306(5704):208I-2084.

26. [http://asrp.cgrb.oregonstate.edu/db]

27. Rattray AJ, McGill CB, Shafer BK, Strathern JN: Fidelity of Mitotic Double-Strand-Break Repair in Saccharomyces cerevisiae: A Role for SAE2/COMI. Genetics 200I, I 58(I): I09-I 22.

28. Cooper DN, Krawczak M: Human Gene Mutation. Oxford, Bios Scientific Publishers; 1993.

29. Wright SI, Agrawal N, Bureau TE: Effects of Recombination Rate and Gene Density on Transposable Element Distributions in Arabidopsis thaliana. Genome Res 2003, I3(8): |897-1903.

30. Zhang L, Gaut BS: Does Recombination Shape the Distribution and Evolution of Tandemly Arrayed Genes (TAGs) in the Arabidopsis thaliana Genome? Genome Res 2003 I 3( I 2):2533-2540.

31. Nordborg $M, H u$ TT, Ishino $Y$, Jhaveri J, Toomajian $C$, Zheng $H$ Bakker E, Calabrese P, Gladstone J, Goyal R, Jakobsson M, Kim S, 
Morozov Y, Padhukasahasram B, Plagnol V, Rosenberg NA, Shah C, Wall JD, Wang J, Zhao K, Kalbfleisch T, Schulz V, Kreitman M, Bergelson J: The Pattern of Polymorphism in Arabidopsis thaliana. PLoS Biology 2005, 3(7):el 96.

32. Nachman MW, Crowell SL: Estimate of the Mutation Rate per Nucleotide in Humans. Genetics 2000, I56(I):297-304.

33. Martinez-Arias R, Calafell F, Mateu E, Comas D, Andres A, Bertranpetit J: Sequence Variability of a Human Pseudogene. Genome Res 200I, I I (6): I07|-I085

34. Bergstrom TF, Erlandsron R, Engkvist H, Josefsson A, Erlich HA, Gyllensten U: Phylogenetic history of hominoid DRB loci and alleles inferred from intron sequences. Immunological Reviews 1999 , 167(I):35I-365.

35. Chen FC, Vallender EJ, Wang H, Tzeng CS, Li WH: Genomic Divergence Between Human and Chimpanzee Estimated from Large-Scale Alignments of Genomic Sequences. J Hered 200 I, 92(6):48I-489.

36. Keightley PD, Eyre-Walker A: Deleterious Mutations and the Evolution of Sex. Science 2000, 290(5490):33I-333.

37. Duret L, Semon M, Piganeau G, Mouchiroud D, Galtier N: Vanishing GC-Rich Isochores in Mammalian Genomes. Genetics 2002, I 62(4): I 837-1847.

38. Kumar S, Subramanian S: Mutation rates in mammalian genomes. PNAS 2002, 99(2):803-808.

39. Subramanian S, Kumar S: Neutral Substitutions Occur at a Faster Rate in Exons Than in Noncoding DNA in Primate Genomes. Genome Res 2003, 13(5):838-844.

40. Yang Z: Among-site rate variation and its impact on phylogenetic analyses. Trends in Ecology \& Evolution 1996, I I (9):367-372.

4I. Bennetzen JL: Transposable element contributions to plant gene and genome evolution. Plant Molecular Biology 2000, V42(I):25I-269.

42. Morton BR, Wright SI: Selective Constraints on Codon Usage of Nuclear Genes from Arabidopsis thaliana. Mol Biol Evol 2007, 24: 122-9.

43. Rattray AJ, Strathern JN: Error-prone DNA Polymerases: When Making a Mistake is the Only Way to Get Ahead. Annua Review of Genetics 2003, 37(I):31-66.

44. Wright SI, Foxe JP, DeRose Wilson L, Kawabe A, Looseley M, Gaut $B$, Charlesworth D: Testing for effects of recombination rate on nucleotide diversity in natural populations of Arabidopsis lyrata. Genetics 2006:genetics. 106.062588

45. Gaut BS, Wright SI, Rizzon C, Dvorak J, Anderson LK: Recombination: An underappreciated factor in the evolution of plant genomes. Nature Genetics 2007, in press:.

46. Kuittinen $H$, de Haan AA, Vogl C, Oikarinen S, Leppala J, Koch M, Mitchell-Olds T, Langley CH, Savolainen O: Comparing the Linkage Maps of the Close Relatives Arabidopsis lyrata and A. thaliana. Genetics 2004, I 68(3): I 575-I584.

47. Ellegren H, Smith NGC, Webster MT: Mutation rate variation in the mammalian genome. Current Opinion in Genetics \& Development 2003, I3(6):562-568.

48. Pesole G, Dellisanti G, Preparata G, Saccone C: The importance of base composition in the correct assessment of genetic distance. Journal of Molecular Evolution 1995, 4 I(6): I I 24- I I 27.

49. Castresana J: Genes on human chromosome I 9 show extreme divergence from the mouse orthologs and a high GC content. Nucl Acids Res 2002, 30(8): 175 I- 1756.

50. Webster MT, Axelsson E, Ellegren H: Strong Regional Biases in Nucleotide Substitution in the Chicken Genome. Mol Biol Evol 2006, 23(6): $1203-1216$

5I. Mitchell-Olds T, Schmitt J: Genetic mechanisms and evolutionary significance of natural variation in Arabidopsis. Nature 2006, 44I(7096):947-952.

52. Le QH, Wright S, Yu Z, Bureau T: Transposon diversity in Arabidopsis thaliana. PNAS 2000, 97(13):7376-738I.

53. Altschul SF, Madden TL, Schaffer AA, Zhang J, Zhang Z, Miller W, Lipman DJ: Gapped BLAST and PSI-BLAST: A new generation of protein database search programs. Nucleic Acids Research 1997, 25:3389-3402.

54. Nickerson DA, Tobe VO, Taylor SL: PolyPhred: automating the detection and genotyping of single nucleotide substitutions using fluorescence-based resequencing. Nucl Acids Res 1997, 25(I4):2745-275I.

55. Thompson JD, Higgins DG, Gibson T]: CLUSTAL W: improving the sensitivity of progressive multiple sequence alignment through sequence weighting, position-specific gap penalties and weight matrix choice. Nucleic Acids Research 1994, 22(22):4673-4680.

56. Wang J, Keightley P, Johnson T: MCALIGN2: Faster, accurate global pairwise alignment of non-coding DNA sequences based on explicit models of indel evolution. BMC Bioinformatics 2006, 7( I):292.

57. [http://titus.bio.uci.edu/data.htm].

58. Kumar S, Tamura K, Jakobsen IB, Nei M: MEGA2: molecular evolutionary genetics analysis software 10.1093/bioinformatics/ 17.12.1 244. Bioinformatics 200I, I 7(12): 1244-1245.
Publish with Biomed Central and every scientist can read your work free of charge

"BioMed Central will be the most significant development for disseminating the results of biomedical research in our lifetime. "

Sir Paul Nurse, Cancer Research UK

Your research papers will be:

- available free of charge to the entire biomedical community

- peer reviewed and published immediately upon acceptance

- cited in PubMed and archived on PubMed Central

- yours - you keep the copyright
BioMedcentral 